hep-th/0312014

\title{
Holography, the Cosmological Constant and the Upper Limit of the Number of e-foldings
}

\author{
Rong-Gen Cai* \\ Institute of Theoretical Physics, Chinese Academy of Sciences, \\ P.O. Box 2735, Beijing 100080, China
}

\begin{abstract}
If the source of the current accelerating expansion of the universe is a positive cosmological constant, Banks and Fischler argued that there exists an upper limit of the total number of e-foldings of inflation. We further elaborate on the upper limit in the senses of viewing the cosmological horizon as the boundary of a cavity and of the holographic Dbound in a de Sitter space. Assuming a simple evolution model of inflation, we obtain an expression of the upper limit in terms of the cosmological constant, the energy density of inflaton when the inflation starts, the energy density as the inflation ends, and reheating temperature. We discuss how the upper limit is modified in the different evolution models of the universe. The holographic D-bound gives more high upper limit than the entropy threshold in the cavity. For the most extremal case where the initial energy density of inflation is as high as the Planck energy, and the reheating temperature is as low as the energy scale of nucleosynthesis, the D-bound gives the upper limit as 146 and the entropy threshold as 122. For reasonable assumption in the simplest cosmology, the holographic D-bound leads to a value about 85, while the cavity model gives a value around 65 for the upper limit, which is close to the value in order to solve the flatness problem and horizon problem in the hot big bang cosmology.
\end{abstract}

*e-mail address: cairg@itp.ac.cn 


\section{Introduction}

The holographic principle is perhaps one of most fundamental principles of nature [1]. This principle relates a theory including gravity in $D$ dimensions to a theory without gravity in lower dimensions. The AdS/CFT correspondence [2] is a beautiful example of the realization of the holographic principle in an anti-de Sitter (AdS) space. Two years ago, Strominger [3] argued that a similar correspondence (dS/CFT correspondence) may also exist in a de Sitter space, which is a maximally symmetric curved space of positive constant curvature.

Needless to say, the de Sitter space plays an important role in modern cosmology. In the inflation model the universe is in a quasi-de Sitter phase. Furthermore, a lot of astronomical observations [4, 5, 6, indicate that the expansion of our universe is now accelerating, rather than decelerating. A most simple explanation of the observed accelerating is that our universe has a positive cosmological constant with the value $\left(\Lambda_{0} \sim\left(10^{-3} e v\right)^{4}\right)[6]$. In that case, out universe will approach again to a de Sitter phase in the far future.

The inflation model [7, 8, 9, which says that our universe has undergone an accelerating epoch in the early time, is now a popular paradigm, in which some fundamental difficulties in the hot big bang cosmology can be solved, for example, the problems of the spatial flatness, the large-scale smoothness, the small-scale inhomogeneity, and the unwanted relics, etc.. A remarkable series of observations support the inflation model (for example, see [10]). It is then natural to ask whether the holography principle has any consequence to the inflation model. In Ref. [1] Albrecht, Kaloper and Song have argued that the holography could lead to a nontrivial energy scale (UV cutoff) above which the description of the effective field theory for perturbations during the inflation losses its validness. However, it results in a series of controversies [12] the D-bound could not give us a nontrivial UV cutoff; instead it is satisfied naturally. The so-called D-bound [13] says that there is a maximal entropy bound of a system in a de Sitter space; the bound is given by the difference between the cosmological horizon areas of pure de Sitter space and asymptotically de Sitter space containing the system.

On the other hand, it is well known that in order to solve the spatial flatness problem and the horizon puzzle, there is a low limit of the number of e-foldings of inflation [7, 8]. Quite interestingly it has been shown robustly that there is also an upper limit for the number $(\tilde{N})$ of e-foldings before the end of inflation at which observable perturbations were generated [14, 15]. However, it says nothing about the total number $(N)$ of e-foldings of inflation, which is usually a much large number than $\tilde{N}$. If the current acceleration is due to a cosmological constant, very recently Banks and Fischler [16] have argued that 
there exists an upper limit of the total number $(N)$ of e-foldings of inflation, $e^{N}=a_{e} / a_{i}$. Here $a_{e}$ and $a_{i}$ denote the scale factors of the universe at the end of inflation and at the beginning of inflation, respectively. Obviously both the numbers $(\tilde{N}$ and $N)$ of e-foldings of inflation play a crucial role in building a successful inflation model, which is still lacking, and in understanding the evolution of the universe. In the brane world scenario, a similar upper limit has been discussed in Ref. [17].

In this note we would like to discuss further the upper limit of the total number of efoldings of inflation. In the next section, we first review the Banks-Fischler's limit briefly. With a simple assumption of evolution of the universe, we then obtain an expression of the upper limit in terms of the cosmological constant, the initial energy density and end energy density during inflation, and the reheating temperature, and discuss some subtleties of the upper limit. In Sec. 3 we use the D-bound to give the upper limit of the total number of e-foldings and discuss some related points. The conclusion is included in Sec. 4 .

\section{Banks-Fischler's Limit}

The key point of inflation to solve the spatial flatness problem and the horizon puzzle is the production of a big amount of entropy during reheating after the end of inflation [7]. The starting point to get the upper limit of the total number of e-foldings is also the entropy in the universe.

For a fluid with an equation of state, $p=\kappa \rho$, contained in a finite cavity with radius $R$, the authors of Ref. [18] have shown that there is an upper bound of entropy stored in the fluid, beyond which black holes will form. The upper bound has the scaling relation to the radius

$$
S_{R} \sim R^{3-2 /(1+\kappa)}
$$

This relation holds in four dimensions ${ }^{1}$. On the other hand, a static observer in a de Sitter space with cosmological constant, $\Lambda_{0}$, cannot see the whole space, the boundary of the region which can be causally accessible to the observer is called the cosmological horizon with size $R_{c} \sim 1 / \sqrt{\Lambda_{0}}$. Banks and Fischler [16] argued that the cosmological horizon could be the natural boundary of the cavity with radius $R_{c}$. Then the threshold

\footnotetext{
${ }^{1}$ In $(n+1)$-dimensions we find that the upper bound of entropy should have the form $S_{R} \sim$ $R^{n-(n-1) /(1+\kappa)}$.
} 
of entropy of the fluid that is accessible to the static observer in the de Sitter space is ${ }^{2}$

$$
S_{C} \sim \Lambda_{0}^{-\frac{1+3 \kappa}{2(1+\kappa)}}
$$

It is interesting to note that in order to avoid the big crush for a closed FriedmannRobertson-Walker (FRW) universe with a positive cosmological constant, the upper bound of the entropy of the fluid in the universe is also given by (2.2) [18]. Of course, it should be reminded that for a flat or open FRW universe with a positive cosmological constant, one cannot reach this conclusion.

Now let us consider thermodynamics of the universe. The Friedmann equation describing the evolution of a flat FRW universe with a positive cosmological constant, $\Lambda$, is

$$
H^{2}=\rho+\frac{\Lambda}{3}
$$

Using the thermodynamic relation, that relates the energy density to the entropy density of a thermodynamic system,

$$
\sigma \sim \rho^{1 /(1+\kappa)}
$$

one can write the entropy of the fluid in the FRW universe with scale factor $a$ as

$$
S \sim a^{3} \rho^{1 /(1+\kappa)} .
$$

Consider the moment at which the inflation ends, and denote the energy density of the universe by $\rho_{e}$. Suppose that the initial size of the causal patch is given by the Hubble radius at the beginning of inflation, $H_{i}^{-1}$. During inflation this patch grows by a factor of $e^{N}$; the scale factor at the end of inflation is then $a \sim \exp (N) H_{i}^{-1}$. Using (2.5), the total entropy in that patch therefore is

$$
S \sim \exp (3 N) H_{i}^{-3} \rho_{e}^{1 /(1+\kappa)} .
$$

Having considered that the change of the Hubble radius is small during inflation, one can take

$$
\rho_{e} \sim H_{i}^{2} \sim \Lambda_{I}
$$

where $\Lambda_{I}$ is the value of the energy density during inflation. The entropy (2.6) of fluid should be less than the one (2.2), which leads to the Banks-Fischler's limit of the total number of e-foldings [16]

$$
N<\frac{1+3 \kappa}{6(1+\kappa)} \ln \frac{\Lambda_{I}}{\Lambda_{0}} .
$$

\footnotetext{
${ }^{2}$ In some expressions concerning entropy throughout the paper, a numerical factor of order $\mathcal{O}(1)$ has been omitted. Such a factor has a negligible effect to the upper limit since it appears as $\ln \mathcal{O}(1)$ in the expressions of the upper limit. See also [16].
} 
It should be stressed that the universe has been assumed to be asymptotically de Sitter here. Note that the dark energy density has the same order as the critical density, so $\Lambda_{0} \sim\left(10^{-3} e v\right)^{4}$. Suppose that the energy density of inflation is at $\Lambda_{I} \sim\left(10^{16} \mathrm{Gev}\right)^{4}$, one then has

$$
N<85
$$

in the case of $\kappa=1$, for the stiff matter. Such an equation of state appears when the universe is filled with a kinetic energy dominated scalar field. On the other hand, one has

$$
N<65
$$

in the case of $\kappa=1 / 3$, for the radiation matter. It is interesting to note that these values for the total number of e-foldings of inflation are close to the value necessary to solve the horizon problem and flatness problem [16, 7]. However, we find that in the matter dominated phase with $\kappa=0$, for the $\Lambda_{I}$ and $\Lambda_{0}$ given above,

$$
N<43
$$

Note that in the usual inflation model, the inflation is followed by a matter dominated phase where the inflaton is rapidly oscillating about a minimum of its potential [8, 9]. The value (2.11) seems too small and is seemingly excluded according to the current astronomical observations [14, 15].

Of course the above estimation is very rough. In this section we are interested in what the maximal and minimal upper limits of the total number of e-foldings are within possible inflation models, if one insists that the entropy threshold in the asymptotically de Sitter space is given by (2.2). For this end, it seems worth to recall the main evolution processes of the universe [8], in particular, the processes before the radiation dominated phase. In a simple inflation model, for example, the single field model, suppose that the inflation starts with the size $H_{i}^{-1}$ of the causal patch and the energy density $\rho_{i}$, in which the potential of the inflaton dominates, and ends with energy density $\rho_{e}$. During inflation, the causal path expands to $\exp (N) H_{i}^{-1}$. After inflation, the universe goes into a coherent oscillation phase, in which the inflaton is rapidly oscillating around a minimum of its potential. After some time, the inflaton decays rapidly to particles in the standard model and the universe is "reheated". Following the reheating is just the ordinary adiabatic, radiation-dominated phase of the hot big bang cosmology. During the reheating, the universe grows by a factor of $a_{r e} / a_{e} \sim\left(\rho_{e} / T_{r e}^{4}\right)^{1 / 3}$, where $\rho_{e}$ is the energy density at the end of inflation and $T_{r e}$ denotes the reheating temperature, which is the initial temperature of the radiation epoch. When the energy density of radiation matter equals to that of 
nonrelativistic matter, the universe enters into the matter dominated phase. As is well known, the total entropy within a casual patch is conserved in the standard cosmology model. In other words, the current total entropy mainly from the radiation matter of our observable universe is in the same order as that during the radiation dominated phase.

With the above consideration, the total entropy of our universe at the beginning of the radiation dominated phase (at that moment the radiation temperature is just the reheating temperature), is ${ }^{3}$

$$
S \sim \exp (3 N) H_{i}^{-3}\left(\rho_{e} / T_{r e}^{4}\right) T_{r e}^{3}
$$

In this case, the entropy bound $S<S_{C}$ yields

$$
N<\frac{1}{4} \ln \frac{\rho_{i}}{\Lambda_{0}}+\ln \frac{\rho_{i}^{1 / 4}}{T_{r e}}+\frac{4}{3} \ln \frac{T_{r e}}{\rho_{e}^{1 / 4}} .
$$

Comparing (2.8) (take $\kappa=1 / 3$ ) with our result (2.13), it is easy to see that they give the same value only when $\rho_{i} \sim \rho_{e} \sim T_{r e}^{4}$. Further we note that the right hand side of (2.13) can be rewritten as

$$
N<\frac{1}{4} \ln \frac{\rho_{i}}{\Lambda_{0}}+\frac{1}{4} \ln \frac{\rho_{i}}{\rho_{e}}+\frac{1}{12} \ln \frac{T_{r e}^{4}}{\rho_{e}} .
$$

Obviously it is not an easy matter to determine the upper limit of the number of e-foldings of inflation since we are still not yet very clear for many events during evolution of the universe, in particular, for events which happened before nucleosynthesis. In other words, there are a lot of uncertainties to determine the upper limit. So following [15], we now consider how the upper limits of the number of e-foldings is modified as one changes the properties of inflation model within the range allowed by theories and observations.

(1) The extremal case. In the chaotic inflation model [19], the initial energy density could be as high as the Planck scale, $\rho_{i} \sim\left(10^{19} G e v\right)^{4}$. On the other hand, the most extremal assumption could be that the reheating continues almost to the nucleosynthesis, which happens at the energy scale $\left(10^{-3} \mathrm{Gev}\right)^{4}$, though the electroweak scale $\left(10^{2} \mathrm{Gev}\right)^{4}$ is regarded usually as the practical limit at the end of inflation [15]. In addition, in supersymmetric theories in order to avoid the overproduction of gravitinos, the energy density should be below $\left(10^{11} \mathrm{Gev}\right)^{4}$. Due to the red shift of energy during reheating, the reheating temperature is usually less than the energy density at the end of inflation (that is, the third term of the right hand side of equation (2.14) gives a negative contribution to the total number), so the extremal assumption is that the most high reheating temperature

\footnotetext{
${ }^{3}$ It is the entropy of radiation matter. Note that the number of particle species is cancelled here.
} 
is of the order of $\rho_{e}^{1 / 4}$. Consider the three energy scales given above as $\rho_{e}$, we find from (2.14) that

$$
\begin{array}{r}
N \sim 122, \text { for } \rho_{e} \sim\left(10^{-3} \mathrm{Gev}\right)^{4} ; \quad N \sim 110, \quad \text { for } \rho_{e} \sim\left(10^{2} \mathrm{Gev}\right)^{4} \\
\text { and } N \sim 90, \quad \text { for } \rho_{e} \sim\left(10^{11} \mathrm{Gev}\right)^{4}
\end{array}
$$

respectively. Although the differences among the three energy scales are large, the differences of total numbers of e-foldings are not so large as expected.

(2) A plausible upper limit. A plausible energy scale at which the inflation starts is the GUT scale with $\rho_{i} \sim\left(10^{16} \mathrm{Gev}\right)^{4}$. It is well known that during inflation, the potential goes down very slowly. Suppose that the inflation ends at $\rho_{e} \sim\left(10^{14} G e v\right)^{4}$. Although there are still considerable uncertainties for the reheating temperature, the total number of e-foldings is not very sensitive to the third term on the right hand side of equation (2.14) since there is a suppressing factor of $(1 / 12)$ in this term. For example, we have

$$
\begin{aligned}
& N \sim 68, \quad \text { for } T_{r e} \sim 10^{12} \mathrm{Gev} ; \quad N \sim 62, \quad \text { for } T_{r e} \sim 10^{5} \mathrm{Gev} \\
& \text { and } N \sim 56, \quad \text { for } T_{r e} \sim 10^{-3} \mathrm{Gev},
\end{aligned}
$$

respectively. With the given $\rho_{i}$ and $T_{r e}$, a more high energy density $\rho_{e}$ at the end of inflation will lead to a lower upper limits than those given by (2.16). An inflation model with more low energy scale is that the inflation begins with $\rho_{i} \sim\left(10^{14} \mathrm{Gev}\right)^{4}$, which satisfies the constraint of gravity wave amplitude from the observation of CMB anisotropy [14. Suppose the inflation ends with $\rho_{e} \sim\left(10^{12} G e v\right)^{4}$, we then have

$$
\begin{gathered}
N \sim 65, \quad \text { for } T_{r e} \sim 10^{12} \mathrm{Gev} ; \quad N \sim 61, \quad \text { for } T_{r e} \sim 10^{8} \mathrm{Gev} ; \\
\text { and } N \sim 58, \quad \text { for } T_{r e} \sim 1 T e v .
\end{gathered}
$$

These values are obviously close to the minimal value necessary to solve the spatial flatness problem and horizon problem [7, 8].

(3) The model of $\lambda \phi^{4}$. This model is special in the sense that reheating in this model has an unusual feature. Usually the universe is in a matter dominated phase during the scalar field oscillation. For the $\lambda \phi^{4}$ model, however, the expansion of the universe is as radiation dominated [20]. In this case, the duration of the epoch of reheating no longer matters and one can take the universe as radiation dominated beginning at the end of inflation [15]. As a result, the factor $\rho_{e} / T_{r e}^{4}$ in (2.12) is absent. Then we have

$$
N<\frac{1}{4} \ln \frac{\rho_{i}}{\Lambda_{0}}+\frac{1}{4} \ln \frac{\rho_{i}}{T_{r e}^{4}}
$$


During the coherent oscillation phase in the usual inflation models, much of the initial vacuum energy is red shifted away, by a factor of $\rho_{e} / T_{r e}^{4}$. In general $T_{r e}^{4}$ therefore is always less than $\rho_{e}$. In the $\lambda \phi^{4}$ model, due to the absence of the factor $\rho_{e} / T_{r e}^{4}$, we have the conclusion that within the same other conditions (the same initial potential of inflation and the same reheating temperature), the upper limit of the number of e-foldings in the $\lambda \phi^{4}$ model is larger than the usual inflation models, for example, the scalar field model with a quadratic potential.

(4) The stiff matter case. In the literature there are proposals to end inflation by making the inflation field a transition from a potential dominated phase to a kinetic energy dominated phase (see [15] and references therein). The equation of state of a kinetic dominated inflation field is the one of stiff matter with $\kappa=1$. In this era, the universe expands with $a \sim t^{1 / 3}$ and $\rho \sim 1 / a^{6}$. During this epoch, the universe grows by a factor $\left(\rho_{e} / T_{r e}^{4}\right)^{1 / 6}$. Here we have assumed that the stiff matter dominates from the end of inflation to the beginning of radiation dominated phase in the big bang cosmology. In this case, the expression (2.12) should be replaced by

$$
S \sim \exp (3 N) H_{i}^{-3}\left(\rho_{e} / T_{r e}^{4}\right)^{1 / 2} T_{r e}^{3} .
$$

As a result, we get the upper limit of the total number of e-foldings

$$
N<\frac{1}{4} \ln \frac{\rho_{i}}{\Lambda_{0}}+\frac{1}{4} \ln \frac{\rho_{i}}{\rho_{e}}+\frac{1}{12} \ln \frac{\rho_{e}}{T_{r e}^{4}} .
$$

Because $\rho_{e}>T_{r e}^{4}$, comparing (2.20) with (2.14), we see that the upper limit is raised in this evolution model of the universe. In contrast to the simplest cosmology described above, the upper limit in this stiff matter case increases by $1 / 6 \ln \left(\rho_{e} / T_{r e}^{4}\right)$. This value is not large, compared to the first two terms on the side of right hand of (2.20). For example, it is only 1.53 if $\rho_{e} \sim\left(10^{16} \mathrm{Gev}\right)^{4}$ and $T_{r e} \sim 10^{12} \mathrm{Gev}$.

\section{Holographic Limit}

Bekenstein [21] was the first to consider the issue of maximal entropy for a macroscopic system contained in a given region. For a closed system with total energy $E$, which fits in a sphere with radius $R$ in three spatial dimensions, He argued that there exists an upper bound on the entropy of the system, $S_{B} \leq 2 \pi E R$. This bound is often referred to as the Bekenstein bound. This bound is believed to be valid for a system with negligible self-gravity in a flat spacetime. In an asymptotically de Sitter space, based on the fact that the cosmological horizon of any asymptotically de Sitter spaces is always less than 
the one of a pure de Sitter space, Bousso [13] argued that the maximal entropy of a system embedded in a de Sitter space is limited by

$$
S_{D}=\frac{1}{4 G}\left(A_{0}-A\right)
$$

by applying the generalized second law 22] of black hole thermodynamics to the cosmological horizon. Here $A_{0}$ denotes the cosmological horizon area of the pure de Sitter space, while $A$ for the asymptotically de Sitter space. This bound (3.1) is often called the D-bound. Although the D-bound is the counterpart of the Bekenstein bound in de Sitter space [23, 24], it is applicable to a strongly self-gravity system, even including black holes, in de Sitter space.

As shown in the previous section, the Banks-Fischler's limit is obtained on the basis that there exists an entropy threshold for a fluid in a cavity, beyond which black holes will form in the cavity; and that the de Sitter horizon is viewed as the boundary of the cavity. On the one hand, the de Sitter space is certainly different from the cavity. On the other hand, considerable series of evidence indicate that a lot of black holes exist in our universe. As a result the assumption of Banks and Fischler may be too stringent. In this section we use the D-bound to give an upper limit of total number of e-foldings of inflation.

Applying the D-bound to the current universe, one has

$$
S_{D} \sim\left(\Lambda_{0}^{-1}-H_{0}^{-2}\right) \sim \frac{0.3}{\Lambda_{0}}
$$

Here $\Lambda_{0}$ is the cosmological constant as before, $H_{0}$ is the current Hubble constant, and the 0.3 is obtained by the current observations: $\Omega_{M} \sim 0.3$ and $\Omega_{\Lambda} \sim 0.7$. If one uses the D-bound at the beginning of the radiation dominated phase, the D-bound gives

$$
S_{D} \sim \Lambda_{0}^{-1}
$$

since at that moment $H^{-2} \ll \Lambda_{0}^{-1}$. Therefore during evolution of the universe, the Dbound gives us the almost same entropy threshold of matter in the universe.

Within the simple evolution process of inflation described in the previous section, the entropy (2.12) of radiation matter is limited by the D-bound (3.3), which leads to

$$
N<\frac{1}{3} \ln \frac{\rho_{i}}{\Lambda_{0}}+\frac{1}{6} \ln \frac{\rho_{i}}{\rho_{e}}+\frac{1}{6} \ln \frac{m_{p l}^{2} T_{r e}^{2}}{\rho_{e}},
$$

where $m_{p l} \sim 10^{19} \mathrm{Gev}$ is the Planck mass. Note that the main contribution to the upper limit comes from the term $\ln \left(\rho_{i} / \Lambda_{0}\right)$. Therefore, in general the holographic limit (3.4) is 
larger than the Banks-Fischler limit. For instance, in the extremal case, namely the case (1) discussed in the previous section, we have

$$
\begin{array}{r}
N \sim 146, \quad \text { for } \rho_{e} \sim\left(10^{-3} G e v\right)^{4} ; \quad N \sim 134, \quad \text { for } \rho_{e} \sim\left(10^{2} G e v\right)^{4} ; \\
\text { and } N \sim 114, \quad \text { for } \rho_{e} \sim\left(10^{11} \text { Gev }\right)^{4} .
\end{array}
$$

Further, for comparison, we calculate the upper limits in the case of $\rho_{i} \sim\left(10^{16} \mathrm{Gev}\right)^{4}$ and $\rho_{e} \sim\left(10^{14} G e v\right)^{4}$. They are

$$
\begin{aligned}
& N \sim 91, \quad \text { for } T_{r e} \sim 10^{12} \mathrm{Gev} ; \quad N \sim 86, \quad \text { for } T_{r e} \sim 10^{5} \mathrm{Gev} \\
& \text { and } N \sim 80, \quad \text { for } T_{r e} \sim 10^{-3} \mathrm{Gev}
\end{aligned}
$$

respectively. Obviously, as the case of Banks-Fischler's limit, the holographic limit also depends on properties of inflation models and evolution process of the universe.

\section{Conclusion}

The current dark energy scale is $\rho \sim\left(10^{-3} e v\right)^{4}$, which is much low than the inflation energy scale, which is generally believed to be around the GUT scale $\sim\left(10^{16} \mathrm{Gev}\right)^{4}$. Therefore people might think that the dark energy, which enforces the universe to accelerating expand now, has nothing to do with the inflation happened at the very early time. However, if the accelerating expansion is attributed to a positive cosmological constant, it does have something to do with the inflation model and with the fate of the universe. Banks and Fischler argued that there exists an upper limit of the total number of e-foldings of inflation in terms of the energy scale of inflation and the cosmological constant $\Lambda_{0}$, if one views the universe as a cavity with radius $1 / \sqrt{\Lambda_{0}}$. Although there are a lot of uncertainties to determine the upper limit definitely, we further elaborate on and detail the upper limit: within a simple assumption of evolution of the universe, we obtain an expression of the upper limit of the total number of e-foldings of inflation in terms of the cosmological constant, the initial energy density and end energy density during inflation, and the reheating temperature. In the extremal case, where inflation is assumed to happen at the Planck scale and the reheating continues to the energy scale of nucleosynthesis, the cavity model gives us a value about 122 for the upper limit, while the D-bound leads to a more large value 146 . Within a reasonable assumption for energy scales of inflation, the upper limit is around 65, which is close to the value necessary to solve the spatial flatness problem and horizon problem in the hot big bang cosmology. We also discuss how the upper limit is modified when one changes the properties of inflation models. 


\section{Acknowledgments}

The author would like to thank Miao Li, Bin Wang, Dehai Zhang and Xinmin Zhang for useful discussions. This work was supported in part by a grant from Chinese Academy of Sciences, a grant from NSFC, a grant from the Ministry of Education of China, and by the Ministry of Science and Technology of China under grant No. TG1999075401.

\section{References}

[1] G. 't Hooft, arXiv:gr-qc/9310026 L. Susskind, J. Math. Phys. 36, 6377 (1995) arXiv:hep-th/9409089.

[2] J. Maldacena, Adv. Theor. Math. Phys. 2, 231 (1998) [Int. J. Theor. Phys. 38, 1113 (1998)] hep-th/9711200; S. S. Gubser, I. R. Klebanov and A. M. Polyakov, Phys. Lett. B 428, 105 (1998) hep-th/9802109; E. Witten, Adv. Theor. Math. Phys. 2, 253 (1998) hep-th/9802150.

[3] A. Strominger, JHEP 0110, 034 (2001) arXiv:hep-th/0106113.

[4] S. Perlmutter et al. [Supernova Cosmology Project Collaboration], Astrophys. J. 483, 565 (1997) arXiv:astro-ph/9608192.

[5] A. G. Riess et al. [Supernova Search Team Collaboration], Astron. J. 116, 1009 (1998) arXiv:astro-ph/9805201.

[6] D. N. Spergel et al., Astrophys. J. Suppl. 148, 175 (2003) arXiv:astro-ph/0302209.

[7] A. H. Guth, Phys. Rev. D 23, 347 (1981).

[8] E.K. Kolb and M.S. Turner, The Early Universe, Addison-Wesley Publishing Company, 1990.

[9] A.R. Liddle and D.H. Lyth, Cosmological Inflation and Large-Scale Structure, Cambridge University Press, 2000.

[10] H. V. Peiris et al., Astrophys. J. Suppl. 148, 213 (2003) arXiv:astro-ph/0302225.

[11] A. Albrecht, N. Kaloper and Y. S. Song, arXiv:hep-th/0211221.

[12] A. Frolov and L. Kofman, JCAP 0305, 009 (2003) arXiv:hep-th/0212327; U. H. Danielsson, JCAP 0303, 002 (2003) arXiv:hep-th/0301182 ; E. Keski-Vakkuri and M. S. Sloth, JCAP 0308, 001 (2003) arXiv:hep-th/0306070. 
[13] R. Bousso, JHEP 0011, 038 (2000) arXiv:hep-th/0010252.

[14] S. Dodelson and L. Hui, Phys. Rev. Lett. 91, 131301 (2003) arXiv:astro-ph/0305113.

[15] A. R. Liddle and S. M. Leach, Phys. Rev. D 68, 103503 (2003) arXiv:astro-ph/0305263.

[16] T. Banks and W. Fischler, arXiv:astro-ph/0307459.

[17] B. Wang and E. Abdalla, arXiv:hep-th/0308145.

[18] W. Fischler, A. Loewy and S. Paban, JHEP 0309, 024 (2003) arXiv:hep-th/0307031.

[19] A. D. Linde, Phys. Lett. B 129, 177 (1983); A. D. Linde, Rept. Prog. Phys. 47, 925 (1984).

[20] L. H. Ford, Phys. Rev. D 35, 2955 (1987).

[21] J. D. Bekenstein, Phys. Rev. D 23, 287 (1981).

[22] J. D. Bekenstein, Phys. Rev. D 9, 3292 (1974).

[23] R. Bousso, JHEP 0104, 035 (2001) arXiv:hep-th/0012052.

[24] R. G. Cai, Y. S. Myung and N. Ohta, Class. Quant. Grav. 18, 5429 (2001) arXiv:hep-th/0105070. 